\title{
Experiment / simulation integrated shape optimization using variable fidelity Kriging model approach
}

\author{
Wataru YAMAZAKI* \\ *Department of Mechanical Engineering, Nagaoka University of Technology \\ 1603-1 Kamitomioka-machi, Nagaoka, Niigata, 940-2188, Japan \\ E-mail: yamazaki@mech.nagaokaut.ac.jp
}

Received: 28 July 2017; Revised: 15 September 2017; Accepted: 3 October 2017

\begin{abstract}
An attempt of experiment / simulation integrated design optimization is performed for the blade airfoil shape of a vertical axis wind turbine. A variable fidelity Kriging surrogate model approach is utilized in this integrated design optimization, in which a surrogate model is constructed from both high-fidelity and low-fidelity sample points information. In this research, the high and low fidelity performance functions are respectively defined from experimental and numerical evaluation methods. In the numerical evaluation method, 2D steady computational fluid dynamics (CFD) simulations are performed for the evaluation of an airfoil shape. An optimal airfoil shape is explored on the accurate variable fidelity surrogate model which is constructed in 9 design variables space representing various blade airfoil shapes. The validity and effectiveness of the present integrated design optimization are discussed by comparing with another approximate optimal airfoil shape which is obtained only by CFD computations. The optimal design obtained by the developed approach showed $8 \%$ larger performance value in the experimental evaluation compared with the approximate optimal design. More efficient shape optimization can be realized by including the low-fidelity information, whose functional trends are utilized to construct accurate surrogate models.
\end{abstract}

Keywords : Variable fidelity model, Surrogate model, Aerodynamic shape optimization, Vertical axis wind turbine, Computational fluid dynamics

\section{Introduction}

Recently, variable fidelity (VF) surrogate model approaches are watched with keen interest, which can construct a surrogate model using both high and low fidelity performance data. Simply stated, an accurate surrogate model can be constructed in this approach using the absolute values of high-fidelity data as well as the functional trends of low-fidelity data as schematically shown in Fig. 1. In the field of computational fluid dynamics (CFD), it is easily possible to define the high and low-fidelity functions from the fidelity difference of physical models (e.g. viscous and inviscid simulations), difference of computational mesh resolution (i.e. fine and coarse mesh evaluations) and so on. The investigations of the variable fidelity approaches are, therefore, one of the most active topics for efficient aerodynamic shape optimization as well as for accurate uncertainty quantification (Kennedy and O'Hagan, 2000, Forrester et al., 2008, Han et al., 2010, Yamazaki and Kuchi-ishi, 2012, Yamazaki and Mavriplis, 2013). According to the reference of Yamazaki and Mavriplis, 2013, an efficient aerodynamic shape optimization has been achieved with a variable fidelity Kriging model approach in which the high and low-fidelity functions are defined with computational mesh resolution. An accurate surrogate model has been also constructed in parameter space using experimental fluid dynamics (EFD) data (as high-fidelity data) and CFD simulation data (as low-fidelity data) according to the reference of Yamazaki and Kuchi-ishi, 2012.

Wind power generation has attracted attention in recent years with the increasing demand for renewable energy. Small wind turbines are watched with keen interest since these are useful for the construction of distributed power generation system and for emergency power source. It is well known that there are two main types of the wind turbines, that are the horizontal-axis wind turbine (HAWT) and vertical-axis wind turbine (VAWT). The former is the more 
conventional wind turbine whose axis of rotation is parallel to the main flow. The HAWT has been the main subject of wind turbine researches for decades. The latter has attracted much more attention in recent years, whose axis of rotation is perpendicular to the main flow. Many experimental/numerical investigations have been performed in the literature (Seki, 1991, Ferreira et al., 2009, Howell et al., 2010, Carrigan et al., 2012, Greenblatt et al., 2012, Bah et al., 2013, Wakashima, et al., 2013, Firdaus et al., 2015, Yamazaki and Arakawa, 2015). The VAWT has an advantage over the HAWT in the flexibility about the direction of the main flow. Lower noise and safety advantage at gust conditions are also pointed out in the literature. In this research, we focus on the Darrieus-type VAWT which generates power due to the lift force produced by rotating blade airfoils.

For better performance of the Darrieus-type VAWT, its airfoil shape design is very important. The flowfield around the Darrieus-type VAWT is complicated unsteady flow with separation around the airfoils. Although CFD methods have reached a high level of maturity in terms of efficiency and robustness in the past years, unsteady CFD evaluations around VAWT configurations are still computationally expensive, especially when its shape optimization is taken into consideration. This is because at least one hundred times of performance evaluation for different airfoil shapes are essential for such airfoil shape optimizations even with state-of-the-art global optimization methods. For this issue of VAWT airfoil design, an efficient shape optimization method for VAWT airfoil has been proposed by the present authors in which the performance is approximately evaluated only by two-dimensional steady flow simulations around an airfoil (Yamazaki and Arakawa, 2015).

In the present research, experiment / simulation integrated shape optimization of VAWT airfoil is investigated using the variable fidelity model approach. Experimental performance evaluations of VAWT are considered as the high-fidelity evaluations while the approximate CFD evaluations are considered as the low-fidelity evaluations. Its validity and effectiveness are discussed in this research. The present paper is organized as follows. The performance evaluation methods used in this research are concisely described in section 2 . In section 3 , the variable fidelity model approach is also concisely described. In section 4, the design optimization problem is defined. In section 5, optimization results obtained only by the approximate CFD evaluations are discussed while the experiment / simulation integrated optimization results are discussed in section 6. Finally, concluding remarks are provided in section 7.

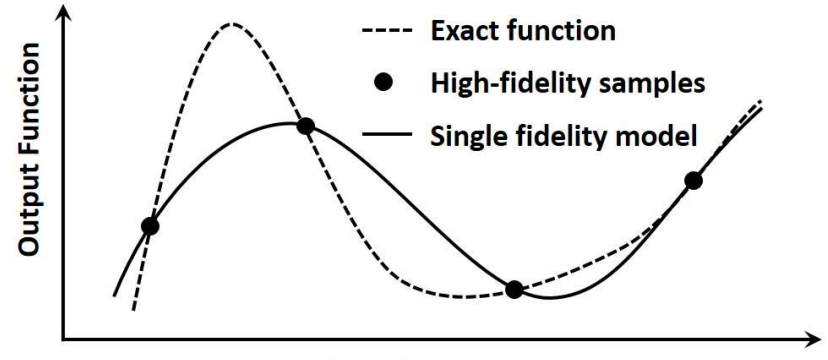

Input Parameter

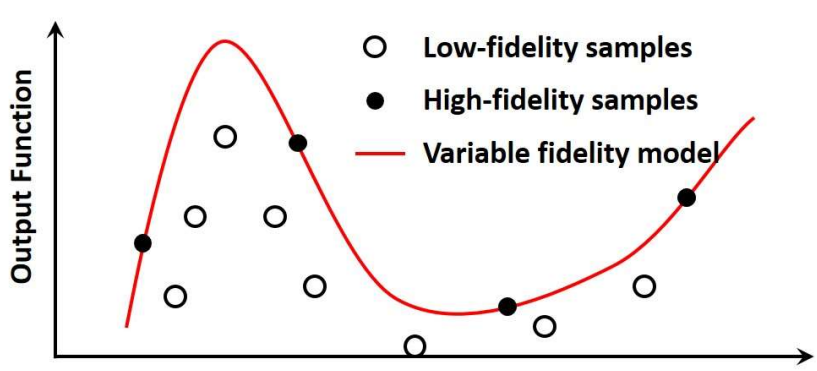

Input Parameter

Fig. 1 Schematic sketches of single fidelity (conventional) surrogate model (left) and variable fidelity surrogate model (right) in one-dimensional design variable space.

\section{Performance evaluation methods}

As the low-fidelity evaluation method, an approximate performance evaluation method of Yamazaki and Arakawa, 2015 is utilized. In this method, the apparent uniform flow magnitude and apparent angle of attack for an airfoil are calculated at arbitral rotational angle $\theta$ from predefined uniform flow magnitude and rotation number. 36 two-dimensional steady flow simulations of an airfoil are performed at ten degrees intervals of the rotational angle with the apparent flow conditions. A torque coefficient $P$ is evaluated at each condition, and then the approximate mean torque coefficient $\bar{P}$ for one rotation is evaluated from the 36 computational results. The schematic sketch of this method is shown in Fig. 2. In this research, the two-dimensional incompressible steady flow simulations are performed by a SIMPLE-based scheme. Since the turbulent intensity of natural wind is generally $10-20 \%$, fully turbulent flows are assumed and the Spalart-Allmaras one equation turbulence model is utilized in the present computations. The computational grid topology utilized in this study is O-type, whose total number of grid points is about 30,000 . Although the calculation cost of this evaluation method is small, it is important to note that there are some assumptions 
in this method as two dimensional steady flows and no consideration about the flow interactions between blade airfoils.

As the high-fidelity evaluation method, on the other hand, a straightforward experimental performance evaluation is performed. Given airfoil shapes are manufactured by the high-resolution 3D printer of Agilista 3100. The dimension of a blade airfoil is $88 \mathrm{~mm}$ (chord length) $\times 200 \mathrm{~mm}$ (blade span length). The printed blade airfoils are indicated in Fig. 3. Since the print resolution (one layer thickness) of the $3 \mathrm{D}$ printer is 15 micro-meter, its surface roughness can be considered to have a negligible effect on the flowfield. Three blades are assembled to make a VAWT rotor whose swept area is $0.04 \mathrm{~m}^{2}$, which is also shown in Fig. 3. The dynamic torque and rotation number are measured by a torque meter of Unipulse UTM- $0.3 \mathrm{Nm}$ by averaging for one minute. The rotation number is controlled by an electromagnetic brake to evaluate the power coefficient curve of the VAWT object. The overview of the developed experimental apparatus is also shown in Fig. 3. The power coefficient of VAWT $C_{P}$ is evaluated as

$$
C_{P}=\frac{T \omega}{\frac{1}{2} \rho A V^{3}}
$$

where $T, \omega, \rho, A, V$ are respectively dynamic torque, angular velocity, air density, swept area of VAWT and wind velocity magnitude.

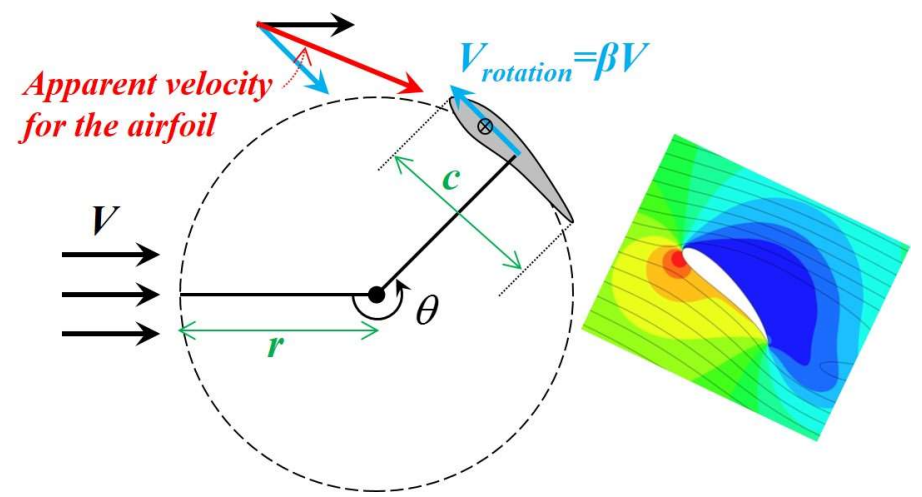

Fig. 2 Schematic sketch of approximate performance evaluation method. Steady flow simulations around an airfoil are performed with apparent flow conditions to evaluate the approximate mean torque coefficient, which corresponds to low-fidelity performance evaluation.
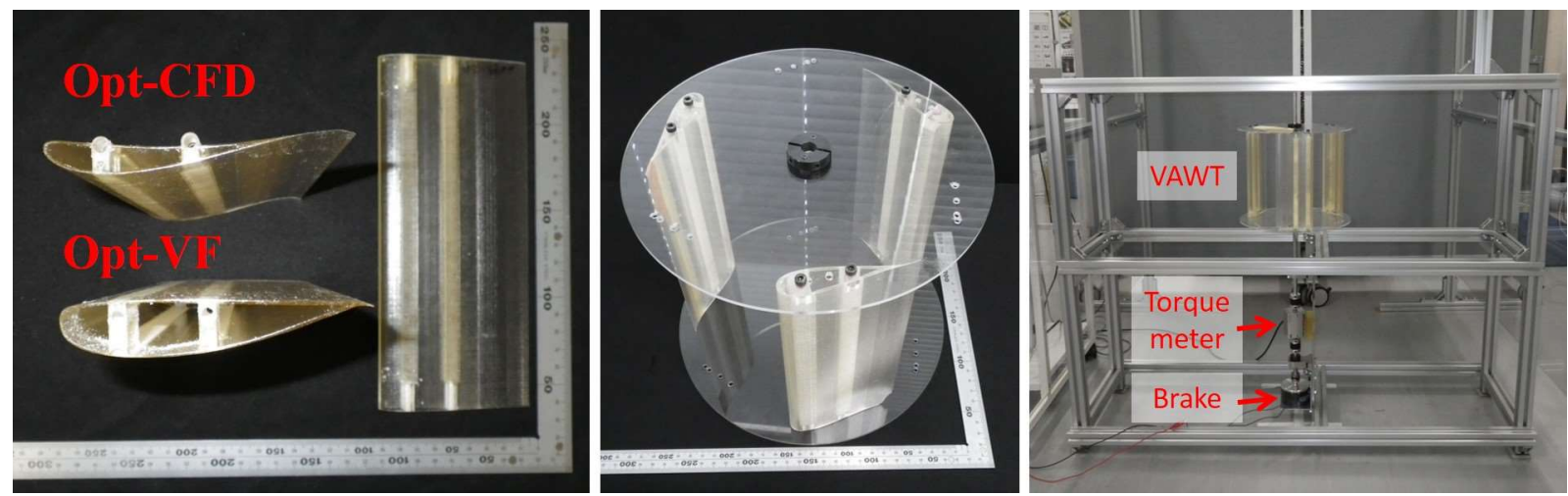

Fig. 3 Overview of experimental performance evaluation, which corresponds to high-fidelity performance evaluation. Left: Printed blade airfoils, Center: VAWT rotor via three blades, Right: Experimental apparatus.

\section{Optimization method}

In this research, a surrogate model-based global design optimization method is utilized which makes use of a Kriging surrogate model. A variable fidelity Kriging surrogate model (Yamazaki and Mavriplis, 2013) is used to construct the surrogate model of a performance function in design variable space using both information of high and low-fidelity sample points. The approximate model of the high-fidelity function is expressed by the weighted sum of the functional values on the high and low-fidelity sample points. The weight coefficients are determined with the 
minimization of the mean square prediction error. In the present variable fidelity approach, the ratio of standard deviation between high and low fidelity functions is determined by the maximum likelihood estimation, and then the (trends of) functional values on low-fidelity samples are utilized to increase the accuracy of the surrogate model of the high-fidelity function. More details are given in Yamazaki and Mavriplis, 2013. Since the present variable fidelity model approach is an extension method of conventional Kriging model approaches as Jones et al., 1998, its optimization procedure is equivalent with that with the conventional Kriging model. Firstly, initial sample points are generated in the design variable space randomly, and then the performance values on the points are evaluated. After the construction of the variable fidelity Kriging model, the search of a promising location in the design variable space is executed by a real-coded genetic algorithm (Fonseca and Fleming, 1993, Kim and Liou, 2009) on the surrogate model. The promising location is explored by the criteria of expected improvement (EI) (Jones et al., 1998) in this research. The function of EI expresses a potential for improvement in design variable space which considers both estimated function value as well as uncertainty of the surrogate model. Additional performance evaluation is performed for the explored promising location (configuration) where EI is maximal, and then new surrogate model is created by adding its information. By the iterative process described above, the accuracy of the surrogate model is efficiently increased around the promising locations in the design variable space. The flowchart of this optimization process is shown in Fig. 4.

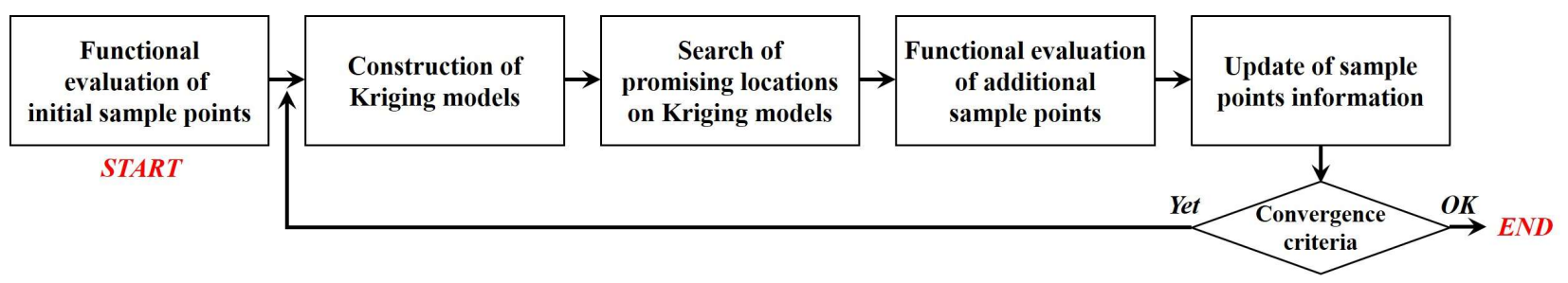

Fig. 4 Flowchart of utilized optimization process using Kriging surrogate model.

\section{Definition of design optimization problem}

In this study, the uniform flow velocity magnitude is set to $7.5 \mathrm{~m} / \mathrm{s}$. The radius of VAWT and airfoil chord length are respectively set to $0.1 \mathrm{~m}$ and $0.088 \mathrm{~m}$. The rotational condition of the blade airfoil is set to $500 \mathrm{rpm}$, which results in the speed ratio (rotational speed over uniform flow speed) $\beta$ of 0.7. The size of the VAWT was specified to have the same size with the experimental apparatus. Various airfoil shapes are defined by Bezier curves as shown in Fig. 5. The upper / lower surfaces are separately defined by two Bezier curves that are based on seven control points. Since the vertical coordinates of the control points (except the fixed points at the leading / trailing edges) are treated as design variables, the total number of design variables is nine in this study, that are defined as Y1-Y9 in Fig. 5. The definition of Y9 is different from the other design variables, which is to satisfy positive thickness of the airfoil at the trailing edge region. Specified ranges of the design variables are summarized in Table 1.

Table 1 Ranges of nine design variables

\begin{tabular}{ccc}
\hline Design Variable & Lower Limit & Upper Limit \\
\hline Y1 & 0.01 & 0.10 \\
\hline Y2 & -0.15 & 0.00 \\
\hline Y3 & -0.15 & 0.05 \\
\hline Y4 & -0.15 & 0.05 \\
\hline Y5 & -0.10 & 0.05 \\
\hline Y6 & 0.00 & 0.15 \\
\hline Y7 & -0.05 & 0.15 \\
\hline Y8 & -0.05 & 0.15 \\
\hline Y9 & 0.02 & 0.10
\end{tabular}




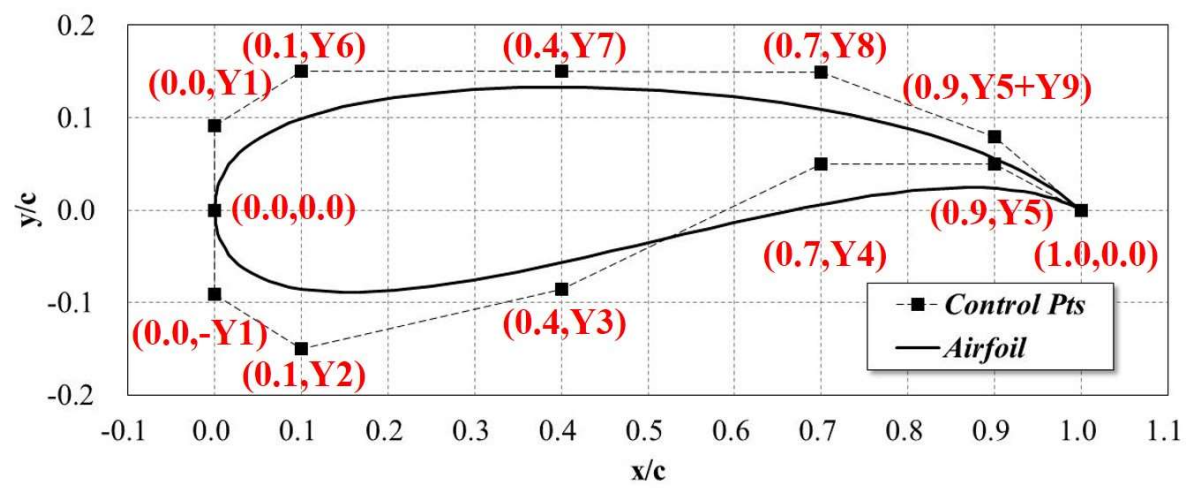

Fig. 5 Airfoil shape parameterization via Bezier curves, in which nine design variables are utilized as Y1-Y9.

\section{Shape optimization only with CFD simulations}

Firstly, the shape optimization result only with CFD simulations is discussed. The number of initial sample points is set to 98 in which 96 samples are generated by the Latin Hypercube sampling. Remaining two samples correspond to NACA642a015 and ClarkY airfoils that are expressed in the design variables space approximately. Then, 50 additional sample points are iteratively selected/evaluated to maximize the approximate mean torque coefficient $\bar{P}$. The obtained optimal design is referred to as Opt-CFD hereafter.

The airfoil shape of Opt-CFD is shown in Fig. 6. It is a negative camber airfoil with very thin thickness at the trailing edge. The performance distribution for one rotation is shown in Fig. 7, whose average value for one rotation corresponds to $\bar{P}$. In the Opt-CFD airfoil, the increase in performance in the range of $270<\theta<360$ degrees is remarkable compared with the existing airfoils. The flowfields at $\theta$ of 250 and 290 degrees are visualized in Fig. 8. The coordinate system in this figure is arranged in such a way that the direction of the apparent uniform flow is horizontally from left to right. At $\theta$ of 290 degrees, the separation region is reduced on the lower surface of the Opt-CFD airfoil, which contributes to the increase in performance. The higher pressure on the upper surface of the Opt-CFD airfoil results in the thrust force component due to the negative camber airfoil shape, which also contributes to the increase in performance.

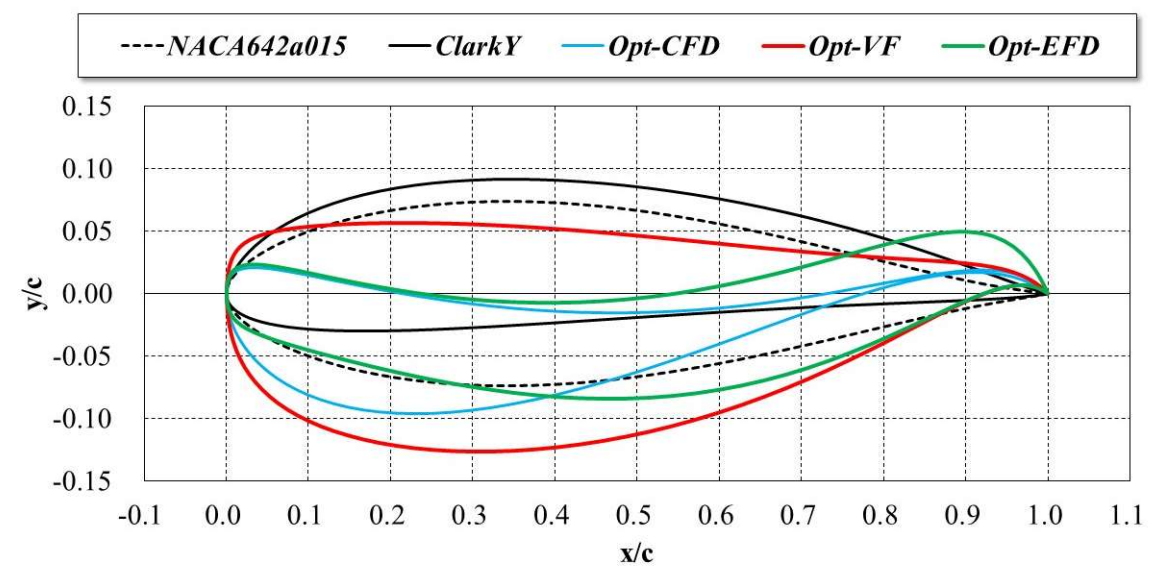

Fig. 6 Comparison of existing airfoils and obtained optimal airfoils. Opt-CFD is the optimal airfoil only using numerical evaluations. Opt-VF is the optimal airfoil via experiment / simulation integrated shape optimization. 


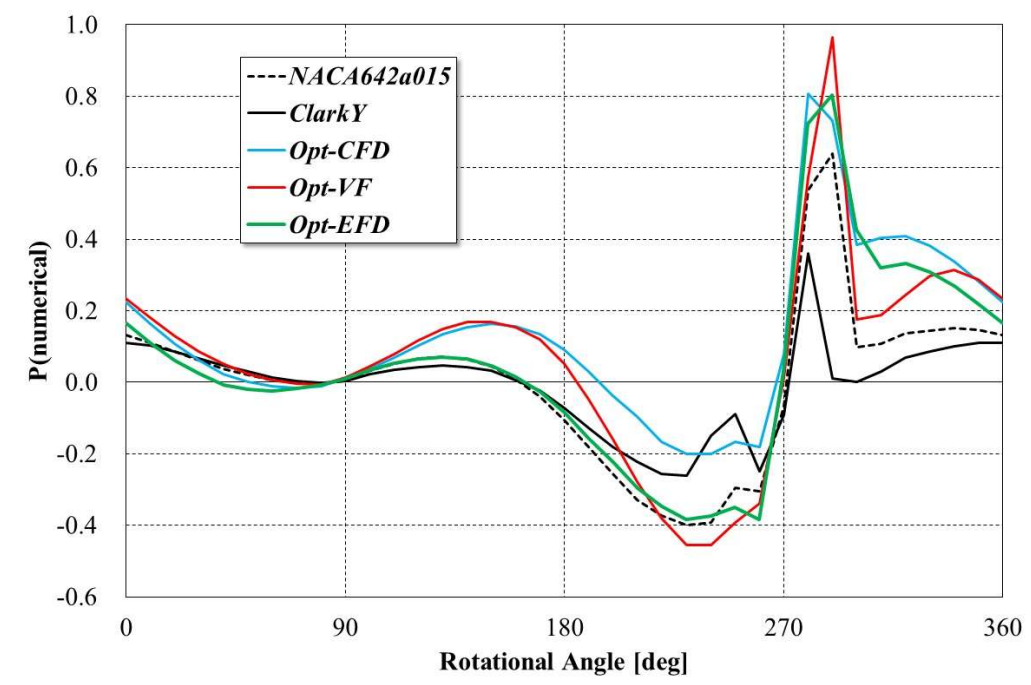

Fig. 7 Comparison of distributions of numerical performance for one rotation. X-axis is the rotational angle $\theta$ defined in Fig. 2.
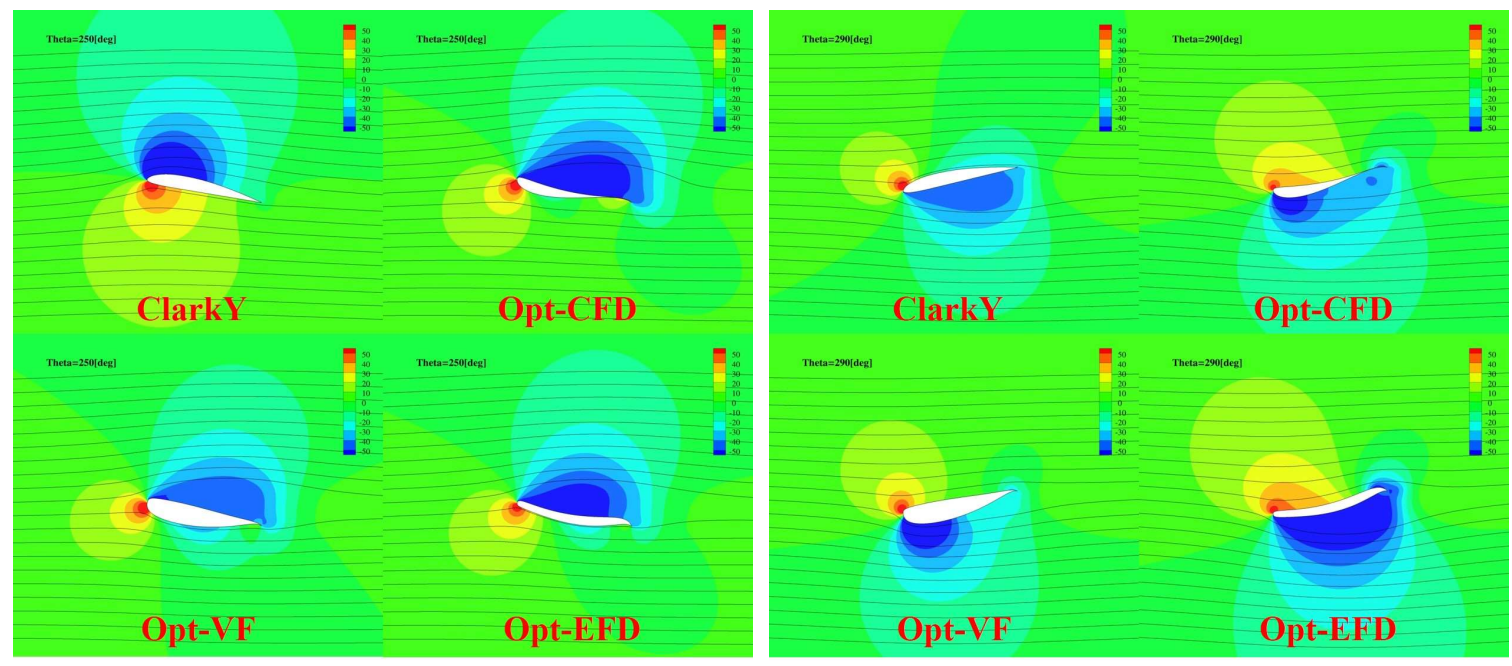

Fig. 8 Flowfield visualizations of representative airfoils. Left: $\theta$ of 250 degrees, Right: $\theta$ of 290 degrees.

\section{Experiment / simulation integrated shape optimization}

In the experiment / simulation integrated shape optimization, the numbers of high and low-fidelity initial sample points are 2 and 98, respectively. The 98 low-fidelity samples are same as that of the previous section. The 2 high-fidelity samples correspond to the NACA642a015 and ClarkY airfoils, which means the implementation of experimental performance evaluations for the two existing airfoils. The initial variable fidelity Kriging model is constructed using these information, and then 6 promising designs are iteratively selected to maximize $C_{P}$. For the promising designs, both fidelity evaluations are performed and then the both performance values are added to update subsequent surrogate models (i.e. two samples are added per promising design). The obtained optimal design, which is sixth promising design, is referred to as Opt-VF hereafter.

The airfoil shape of Opt-VF is included in Fig. 6. Its numerical simulation results are also included in Figs. 7-8. The Opt-VF is also a negative camber airfoil as the Opt-CFD while its airfoil thickness is larger than the Opt-CFD. In the Opt-VF airfoil, the increase in performance in the range of $270<\theta<360$ degrees is confirmed as same as the Opt-CFD. Again, the reduction of separation region can be observed on the lower surface of Opt-VF airfoil at $\theta$ of 290 degrees. The numerical performance of the Opt-VF airfoil is worse than the Opt-CFD especially in the range of $180<\theta<270$ degrees, which results in worse performance value of the Opt-VF airfoil in terms of $\bar{P}$.

Next, the results of experimental performance evaluations are compared. The power coefficient curves are shown in Fig. 9 in which the present design condition corresponds to the speed ratio of 0.7. The Opt-CFD shows better 
performance than the two existing airfoils, which indicates the validity of the approximate performance evaluation method based on two-dimensional steady flow simulations around an airfoil. The Opt-VF shows better performance than the Opt-CFD, which indicates the superiority of the proposed experiment / simulation integration shape optimization approach. The performance values evaluated by the simulation and the experiment are summarized in Table 2. The Opt-VF achieved $8 \%$ increase in the experimental performance compared with the Opt-CFD.

For additional discussion, another optimal airfoil was explored from a (single-fidelity) Kriging model constructed by 7 high-fidelity sample points that were gathered in the experiment / simulation integrated shape optimization ( 2 initial and 5 ( 1 st $\sim 5$ th) promising designs, the Opt-VF was the sixth promising design which was obtained from these 7 high-fidelity samples with 103 low-fidelity samples). This airfoil was obtained only by one cycle of the optimization process, and only from 7 sample points, which implies it is not a decent optimal airfoil in a precise sense. This airfoil was only used for the discussion of the effectiveness of the variable fidelity Kriging model approach, which is referred to as Opt-EFD. The information of the Opt-EFD airfoil is also included in Figs. 6-9 and Table 2. The shape of the Opt-EFD is also a negative camber airfoil and its outline shape is similar with the Opt-CFD. However, its trailing edge is thicker than the Opt-CFD and then its numerical performance value is much worse than the Opt-CFD. The experimental performance value is also much worse than the Opt-CFD and Opt-VF. This is the contrary evidence to the hypothesis that the Opt-VF airfoil was explored just by getting the benefits from the information of the high-fidelity samples (just by changing the objective function from $\bar{P}$ to $C_{P}$ ). It can be concluded that the Opt-VF airfoil was explored not only by getting the benefits from the information of the high-fidelity samples, but also from the functional trends obtained from the low-fidelity samples. Since the low-fidelity evaluation can be executed with smaller cost in general, more efficient shape optimization can be realized in the proposed approach than the conventional (single-fidelity) approach, which can be considered as the advantage of the proposed approach. The relationship between the high and low-fidelity performance values is shown in Fig. 10 in which all designs that were evaluated in the both fidelity functions are included. (Theoretically, $C_{P}$ is proportional to the dynamic torque $T$ at a condition as understood from Eq.(1). Ideally, therefore, $\bar{P}$ will be in linear relationship with $C_{P}$.) We can observe the qualitative correlation between them, and its coefficient of correlation was about 0.7 , which indicates the qualitative validity of the approximate performance evaluation method used as the low-fidelity function.

The Opt-VF airfoil showed better performance than the Opt-CFD in the high-fidelity experimental evaluation while the Opt-CFD showed better performance than the Opt-VF in the low-fidelity numerical evaluation. Here, the reason of this opposite magnitude relationship is presumed. As explained before, there is an assumption of no consideration about flow interactions between the blade airfoils in the low-fidelity evaluation. As shown in Fig. 7, the Opt-CFD has better numerical performance especially in the range of $180<\theta<270$ degrees. An airfoil in the range of $180<\theta<270$ degrees locates in the wake of another precedent airfoil in the experimental evaluation with three blade airfoils, which means it is difficult to accept the assumption in this range of the rotational angle. Thus, it is presumed that the superiority of the Opt-CFD in the low-fidelity numerical evaluation based on the higher performance in the range of $180<\theta<270$ degrees, does not directly appear in the high-fidelity experimental evaluation. On the other hand, the Opt-VF airfoil has taken into account the effect of flow interactions from the high-fidelity functional information given in the variable fidelity Kriging model.

Table 2 Summary of numerical and experimental performance of representative airfoils

\begin{tabular}{ccc}
\hline Airfoil & $\bar{P}$ (numerical) & $C_{P}$ (experimental) \\
\hline NACA642a015 & 0.0001 & 0.115 \\
\hline ClarkY & -0.0077 & 0.084 \\
\hline Opt-CFD & 0.1221 & 0.162 \\
\hline Opt-VF & 0.0644 & 0.175 \\
\hline Opt-EFD & 0.0401 & 0.077 \\
\hline
\end{tabular}




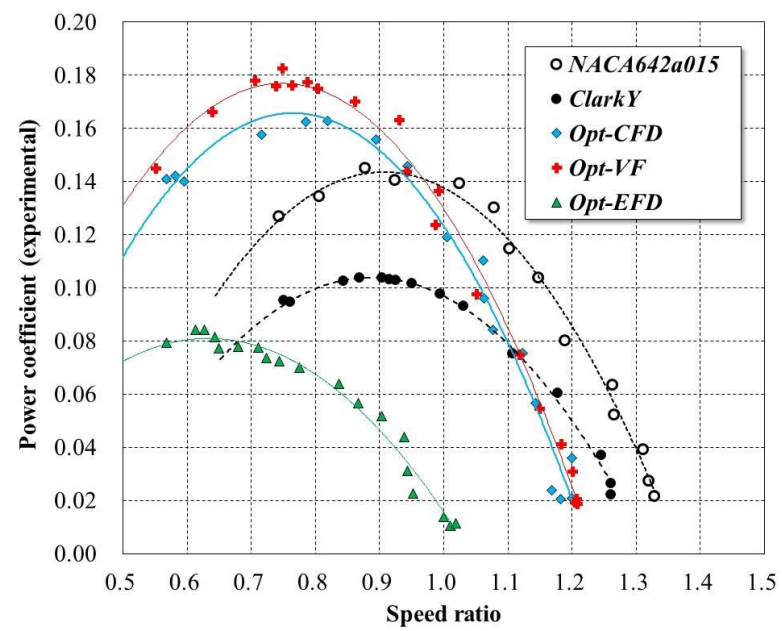

Fig. 9 Comparison of power coefficient curves of representative airfoils evaluated by experiments.

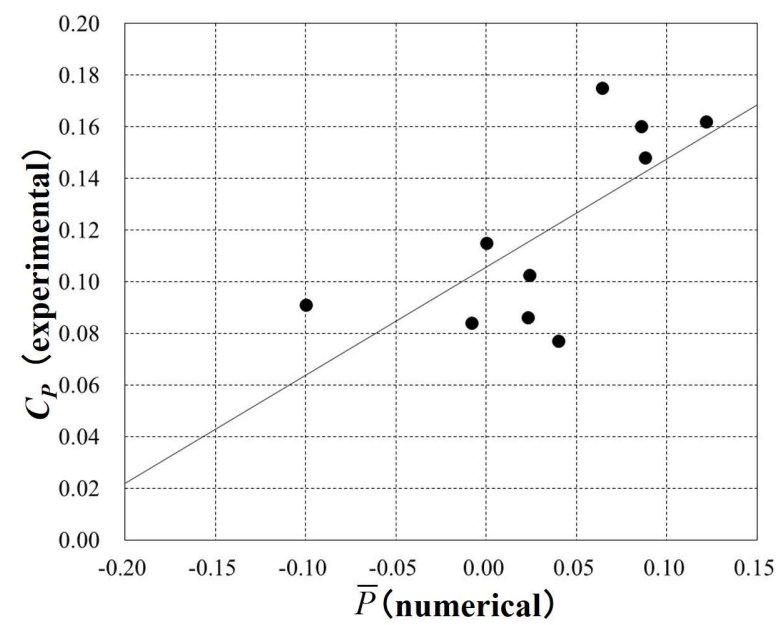

Fig. 10 Relationship between the high (Y-axis) and low (X-axis) fidelity performance evaluations, which indicates the correlation between them.

\section{Conclusions}

In this research, an experiment / simulation integrated shape optimization was investigated using a variable fidelity Kriging model approach. Experimental and numerical performance evaluations were respectively treated as the high and low fidelity functions in this research. With respect to the numerical performance evaluation, there are some assumptions as two dimensional steady flows and no consideration about flow interactions between blade airfoils. The surrogate model of the high-fidelity performance function was constructed using both fidelity information, and then an optimal shape was explored on the surrogate model. The developed approach was applied to a shape optimization problem of VAWT blade airfoil. The optimal airfoils obtained by the developed approach and only by the numerical performance evaluations were compared. Although the both optimal designs were negative camber airfoils, the optimal design by the developed approach was a thicker airfoil. The optimal design by the developed approach showed $8 \%$ larger performance value in the experimental evaluation, which indicated the validity of the developed experiment / simulation integrated shape optimization approach. To demonstrate its practical effectiveness and engineering utility, further investigation will be required considering the design cost of the both fidelity evaluations.

\section{Acknowledgement}

The author is very grateful to Mr. Yuta ARAKAWA, who was a graduate student of Nagaoka University of Technology, for his help in experimental evaluations. A part of this research was supported by JSPS KAKENHI grant 
number 16K21047 as well as Sasaki Environment Technology Foundation. The author is also grateful to the people involved in the high-resolution 3D printer Agilista-3100 which was installed in the innovative creation project via implicit knowledge of Nagaoka University of Technology.

\section{References}

Bah, E. A., Sankar, L., and Jagoda, J. Investigation on the use of multi-element airfoils for improving vertical axis wind turbine performance, AIAA Paper 2013-1109 (2013).

Carrigan, T. J., Dennis, B. H., Han, Z. X., and Wang, B. P., Aerodynamic shape optimization of a vertical-axis wind turbine using differential evolution, ISRN Renewable Energy Volume 2012, Article ID 528418 (2012).

Ferreira, C. S., Kuik, G. V., Bussel, G. V., and Scarano, F., Visualization by PIV of dynamic stall on a vertical axis wind turbine, Experiments in Fluids, Vol.46, Issue 1 (2009), pp.97-108.

Firdaus, R., Kiwata, T., Kono, T., and Nagao, K., Numerical and experimental studies of a small vertical-axis wind turbine with variable-pitch straight blades, Journal of Fluid Science and Technology, Vol.10, No.1 (2015), p.JFST0001.

Fonseca, C. M., and Fleming, P. J., Genetic algorithms for multiobjective optimization: formulation, discussion and generalization, Proceedings of the 5th International Conference on Genetic Algorithms, Morgan Kaufmann Publishers, Inc., San Mateo (1993), pp.416-423.

Forrester, A. I., Sobester, A., and Keane, A. J., Engineering design via surrogate modelling: a practical guide, John-Wiley and Sons (2008).

Greenblatt, D., Schulman, M., and Ben-Harav, A., Vertical axis wind turbine performance enhancement using plasma actuators, Renewable Energy, Vol.37, Issue 1 (2012), pp.345-354.

Han, Z. H., Zimmermann, R., and Görtz, S., A new cokriging method for variable-fidelity surrogate modeling of aerodynamic data, AIAA-2010-1225 (2010).

Howell, R., Qin, N., Edwards, J., and Durrani, N., Wind tunnel and numerical study of a small vertical axis wind turbine, Renewable Energy, Vol.35, Issue 2 (2010), pp.412-422.

Jones, D. R., Schonlau, M., and Welch, W. J., Efficient global optimization of expensive black-box functions, Journal of Global Optimization, Vol.13, Issue 4 (1998), pp.455-492.

Kennedy, M. C., and O'Hagan, A., Predicting the output from a complex computer code when fast approximations are available, Biometrika, Vol.87, No.1 (2000), pp.1-13.

Kim, H. J., and Liou, M. S., New multi-objective genetic algorithms for diversity and convergence enhancement, AIAA Paper 2009-1168 (2009).

Seki, K., Research and development of high-performance airfoil sections for vertical axis wind turbine at low-Reynolds number, Transaction of JSME, Vol.57, No.536 (1991), pp.1297-1304 (in Japanese).

Wakashima, S., Miyashita, Y., Yamada, N., Takahashi, T., Otsuka, Y., and Yamazaki, W., Numerical analysis of a rotating micro straight-wing VAWT model for engineering design educations, Transactions of the Japan Society of Mechanical Engineers Series C Vol.79, No.808 (2013), pp.2562-2566 (in Japanese).

Yamazaki, W., and Arakawa, Y., Inexpensive airfoil shape optimization for vertical axis wind turbine and its validation, Journal of Fluid Science and Technology, Vol.10, No.2 (2015), p.JFST0015.

Yamazaki, W., and Kuchi-ishi, S., A variable fidelity response surface approach towards integration of CFD and EFD, Proceedings of 5th Symposium on Integrating CFD and Experiments in Aerodynamics (2012).

Yamazaki, W., and Mavriplis, D. J., Derivative-enhanced variable fidelity surrogate modeling for aerodynamic functions, AIAA Journal, Vol.51, No.1 (2013), pp.126-137. 